\title{
An adapted optical flow algorithm for robust quantification of cardiac wall motion from standard cine-MR examinations
}

\author{
M. Xavier, A. Lalande, P. M. Walker, F. Brunotte, L. Legrand
}

\begin{abstract}
This paper presents a method for local myocardial motion estimation from a conventional SSFP cine-MRI sequence using a modified phase-based Optical Flow (OF) technique. Initially, the technique was tested on synthetic images to evaluate its robustness with regards to Rician noise and to brightness variations. The method was then applied to cardiac images acquired on 11 healthy subjects. Myocardial velocity is measured in $\mathbf{c m} / \mathrm{s}$ in each studied pixel and visualized as colored vectors superimposed on MRI images. The estimated phase-based OF results were compared with a reference $O F$ method and gave similar results on synthetic images i.e. without a significant difference of the mean Angular Error. Applied on cine-MRI of normal hearts, the calculated velocities from short axis images concord with values obtained in the literature. The advantage of the presented method is its robustness with respect to Rician noise and to brightness changes often observed in cine-MRI sequences, and especially with the through-plane movement of the heart. Motion assessment using our method on cine-MR images gives promising results on motion estimation on a pixel by pixel basis, leading to a regional measurement of the timevelocity course of myocardial displacement in different segments of the heart wall.
\end{abstract} flow

Index Terms - Cine-MRI, heart, motion estimation, optical

\section{INTRODUCTION}

$\mathrm{C}$ ardiac Magnetic Resonance Imaging (MRI) is a noninvasive technique allowing the study of the cardiac function. ECG-gated SSFP (Steady-State Free Precession) cine-MRI sequences have the advantage of providing detailed morphological information coupled with an adequate temporal resolution and an excellent contrast between structures. These sequences are widely used in clinical practice for the study of myocardial function [1] through the estimation of Ejection Fraction (EF) and myocardial thickness.

The evaluation of myocardial motion from a cine-MRI sequence is often limited to the visual evaluation of the regional contractile function [2]. Visual evaluation has the drawback of being subjective and the estimation of the time course of the wall motion remains difficult even for experienced readers. The techniques for cardiac motion

Manuscript received December 16, 2011.

M. Xavier, A. Lalande, P.M. Walker, F. Brunotte and L. Legrand are with the LE2I laboratory, UMR CNRS 6306, Faculté de Médecine, Université de Bourgogne, Dijon, France (corresponding author: Alain Lalande. phone: $(+33)$ 3803933 91; fax: (+33) 3803932 93; e-mail: alain.lalande@ubourgogne.fr). estimation are divided into shape-based [3] and pixel-based approaches. The technique proposed in this paper is based on phase information retrieval by automatically extracting motion information from cardiac cine-MRI using a phase-based Optical Flow (OF) approach. Optical flow is the representation of the apparent two-dimensional (2D) movement of an object in a scene. Considering cardiac MRI sequences, this amounts to computing the apparent in-plane motion field of the heart. This technique allows the detection of the local movement estimated at each pixel in the image. Furthermore, OF may yield sub-pixel precision and does not rely on prior knowledge of image content.

Despite the multitude of methods available for computing OF [4], such techniques are rarely applied to cardiac cine-MRI. As the optical flow techniques assume that brightness gradients are present, one challenge is to take into account the lack of brightness gradients in the relatively homogeneous myocardium. Fortunately, the brightness gradients are usually sufficient on the myocardium, close to the endocardium, due to the contrast between the myocardium and the left ventricle cavity, thus allowing the study of the inner wall of the left ventricle. Due to noise and through-plane movement of the heart causing brightness variations of the image, classical methods based on pixel intensity may fail to estimate motion on time-series of MRI images because the OF constraint equation implies the consistency of the pixel intensity through time. To tackle these problems, we propose to consider the phase-based OF method initially introduced by Fleet and Jepson [5]. The aim of the present study is to develop an adaptation of this algorithm that avoids the aforementioned limitations and reduces the computational cost. The representation of the estimated OF field within an image is commonly delineated by a vectorial motion field superimposed on the image. The vector field represents the local velocity measurements of the various structures within the images. Tracking of points of interest then allows a quantitative analysis of the myocardial displacement through time-radial velocity curves, giving clinicians metrics for the assessment of myocardial wall abnormalities.

\section{METHODOLOGY}

\section{A. Optical flow methods}

Prior works using OF have provided promising results on cine-MRI [6]-[8], on tagged MRI [9]-[11], on computed tomography [12] and echocardiography [13], [14]. In this paper OF methods are evaluated as a way of estimating myocardial motion from cine-MRI data. The order of 
magnitude of the velocities for the left ventricle for healthy adults is between $3 \mathrm{~cm} / \mathrm{s}$ and $6.5 \mathrm{~cm} / \mathrm{s}$, depending on the myocardial segment and the moment of the cardiac cycle (systolic peak velocity or diastolic peak velocity) [13], [15], [16]. OF methods are based on the brightness consistency of pixel intensity as a function of time. So, considering a particular location, a stack of images is acquired over time ( $t$ ) covering the cardiac cycle and a 2D motion field can be estimated on each image. In order to accurately detect motion, the optical flow constraint equation (OFCE) supposes that structure displacements between two consecutive images are small (around 1 or 2 pixels/frame). Then, to ensure reasonable displacements between two consecutive images, the rate of image acquisition was adapted. For example, considering the $10 \mathrm{~ms}$ temporal resolution and the $1.2 \mathrm{~mm}$ pixel spacing, the displacement of 1 pixel between two consecutive frames corresponds to a velocity of $12 \mathrm{~cm} / \mathrm{s}$ (OF techniques provide sub-pixel motion precision). In the following two paragraphs, the Intensity-based OF (IOF) and the Phase-based OF (POF) methods are described. These two methods were chosen as reference methods, despite being slower than others, they were more accurate on the diverging tree sequence (as shown by Liu et al. [17]). Indeed, it is primordial to be accurate when analysing medical image sequences for diagnostic purposes.

Image processing, including pre-processing (noise filtering) and motion estimation along the image sequence, was performed using a program developed under Matlab (MathWorks, Inc) in our laboratory.

\section{Intensity-based methods}

Intensity-based OF (IOF) methods compute image vector fields from spatiotemporal derivatives given by image intensities. Two of these methods, one developed by Horn and Schunck [18] and the other developed by Lucas and Kanade [19] have been tested on MR images by Barron [8] on a preliminary study. This latter approach showed that the quality of the estimated OF directly depends on the quality of the estimated spatio-temporal derivatives, which are very sensitive to image quality. Intensity based methods need the use of an appropriate spatio-temporal smoothness constraint. A global smoothness constraint blurs "motion edges" which appear for example when the heart goes from contraction to dilatation and vice versa.

\section{Phase-based methods}

Phase-based OF (POF) methods for optical flow estimation rely on tracking contours of constant phase over time. The POF technique was initially developed by Fleet and Jepson [5]. They defined component velocity in terms of the instantaneous motion normal to level-phase contours in the output of band-pass velocity tuned filters.

The use of complex Gabor filters that are orientation-sensitive filters in Fourier space allows one to deal with phase information and then, allows an estimation of the instantaneous velocity field. Gabor filters are sinusoidallymodulated Gaussian functions. The response $R(\overrightarrow{\mathbf{x}}, \mathrm{t})$ of a complex Gabor filter can be decomposed into its amplitude $\rho(\overrightarrow{\mathbf{x}}, \mathrm{t})$ and its phase $\phi(\overrightarrow{\mathbf{x}}, \mathrm{t})$ :

$$
R(\overrightarrow{\mathrm{x}}, t)=\rho(\overrightarrow{\mathrm{x}}, t) \cdot \exp (i \phi(\overrightarrow{\mathrm{x}}, t))
$$

The use of a set of filters (filter bank) is motivated by the phase-based optical flow constraint equation (POFCE)

$$
\nabla \phi(\overrightarrow{\mathbf{x}}, t) \cdot V(\overrightarrow{\mathbf{x}}, t)+\phi_{t}(\overrightarrow{\mathbf{x}}, t)=0
$$

where $\nabla \phi=\left[\begin{array}{l}\phi_{x} \\ \phi_{y}\end{array}\right]$ is the spatial phase gradient, $V(\overrightarrow{\mathbf{x}}, \mathrm{t})=\left[\begin{array}{l}v_{x} \\ v_{y}\end{array}\right]$ is the velocity vector to determine, $\phi_{t}$ the temporal phase gradient and $\overrightarrow{\mathbf{x}}$ a pixel at position (x,y). From the POFCE, one can write:

$$
V(\overrightarrow{\mathbf{x}}, t)=\frac{-\phi_{t}(\overrightarrow{\mathbf{x}}, t) \cdot \nabla \phi(\overrightarrow{\mathbf{x}}, t)}{\|\nabla \phi(\overrightarrow{\mathbf{x}}, t)\|^{2}}
$$

where $\|\nabla \phi(\overrightarrow{\mathbf{x}}, t)\|$ is the Euclidean norm of the spatial gradient.

The POFCE only provides the velocity component normal to the contour: this is defined as the aperture problem. For a pixel with $(\mathrm{x}, \mathrm{y}, \mathrm{t})$ coordinates, at least two equations are necessary to get the two-dimensional velocity vector in order to compute the full velocity. The Fleet and Jepson algorithm initially used a complex 3D Gabor filter bank involving a high computational cost [20] and a low OF density (where the OF density is the percentage of non-zero velocity vectors within the image) in spite of the use of separable filters and a reasonably efficient implementation. To counter these flaws, Bruno and Pellerin [21] proposed a new algorithm providing accurate computation of the optical flow between only two frames, without needing spatiotemporal filtering over a large number of frames. They used a spatial Gabor-like filter bank which avoids the aperture problem. The velocity was then robustly estimated with M-estimators. Large and small motion vectors were recovered with a multiresolution scheme. Indeed, to ensure the assessment of both the low and fast velocities, a pyramidal decomposition of images by Gaussian low-pass filtering was considered [22].

The velocity field $V(\overrightarrow{\mathbf{x}}, \mathrm{t})$ was robustly estimated by using a least squares technique in a local neighborhood. Using matrix notations, as Lucas and Kanade [19], one can write:

$$
V=\left[A^{T} W A\right]^{-1} A^{T} W b
$$

where $\quad A=\left[\nabla \phi\left(\overrightarrow{\mathbf{x}}_{1}, t\right), \ldots, \nabla \phi\left(\overrightarrow{\mathbf{x}}_{n}, t\right)\right]^{T}$, $W=\operatorname{diag}\left[W\left(\overrightarrow{\mathbf{x}}_{1}, t\right), \ldots, W\left(\overrightarrow{\mathbf{x}}_{n}, t\right)\right]$ containing the coefficients of a 2D Gaussian function, which reflects the degree of confidence one can have in the estimated speed, and $b=$ $-\left[\phi_{t}\left(\overrightarrow{\mathbf{x}}_{1}, t\right), \ldots, \phi_{t}\left(\overrightarrow{\mathbf{x}}_{n}, t\right)\right]^{T}$. If matrix $A^{T} W A$ is invertible, then matrix $V$ exists.

\section{B. Proposed approach}

\section{Noise filtering}

MR magnitude data are described by a Rician noise distribution [23] in general and by a Rayleigh distribution in the background. This noise comes from complex Gaussian noise in the original frequency domain (k-space). In the case of phase-array coils, data from parallel imaging with GRAPPA (generalized autocalibrating partially parallel imaging acquisition) are followed by an adaptive reconstruction of the complex images of the channel, then the 
noise properties are described by the Rayleigh distribution on the background [24].

Moreover, the probability density function of the MR magnitude image tends towards a Gaussian distribution when the Signal-to-Noise Ratio (SNR) is higher than three [23]. For example, in this study the mean SNR of the myocardium was 10. As a result, MR images are usually pre-smoothed using a Gaussian filter. However, the smoothing step using a Gaussian filter causes blurring within the image and consequently reduces the signal-to-background contrast. For this reason, all magnitude images were pre-filtered using another noise removal algorithm, rather than a Gaussian filter. Several correction schemes have been proposed to estimate the signal intensity from the magnitude MR signal. Among them, an unbiased estimator of the signal intensity that depends on the second moment of the magnitude signal was developed by McGibney and Smith [25]. In the case of a Rician noise, the second-order moment $E\left\{M^{2}\right\}$ of the amplitude $M$ of a measured noisy MR signal can be written

$$
E\left\{M^{2}\right\}=B^{2}+2 \sigma^{2}
$$

where $B$ is the original noiseless MR signal and $\sigma^{2}$ the noise variance. The estimation of the noiseless image is then given by :

$$
\hat{B}=\sqrt{\left\langle M^{2}\right\rangle-2 \sigma^{2}}
$$

It depends on the second order moment computed in a neighborhood $\left\langle M^{2}\right\rangle$, and requires prior knowledge of the noise variance $\sigma^{2}$. This filtering method is based on the assumption that the noise in the background follows a Rayleigh distribution. The noise estimator was computed from the maximum of the histogram of the local first order moment. Therefore, the noise removal algorithm operates from the second order moment of the image and the noise estimator.

\section{Developed optical flow method: the MPOF method}

To improve efficiency and OF density compared with the initial algorithm of Fleet and Jepson, we developed a modified version of the POF algorithm (MPOF) using the spatial filtering introduced by Bruno and Pellerin. Depending on the settings for the acquisition (temporal resolution and pixel size) and the behavior of the heart, using a pyramidal decomposition of images by Gaussian low-pass filtering [22] may be necessary to extend the field of velocities allowed (up to 5 pixels per frame). Here, a three level Gaussian low-pass pyramidal image decomposition was used: Levels 0,1 and 2 respectively cover velocity ranges $0-1.25,1.25-2.5$ and $2.5-$ 5.0 pixels per frame. This ensures the assessment of both the low and fast velocities of the myocardium.

Instead of using complex 3D Gabor filters for estimating optical flow, MPOF consists in a complex 2D spatial Gabor filtering followed by a temporal second-order differentiation. It uses a complex 2D Gabor filter bank made of $N$ filters. Each filter corresponds to a pair of diametrically opposed 2D Gaussians oriented at an angle $\theta_{i}$ and whose impulse response $g_{i}(x, y)$ at the $i$ th filter is:

$$
\mathrm{g}_{i}(x, y)=\frac{1}{2 \pi \sigma^{2}} \mathrm{e}^{-\left(x^{2}+y^{2}\right) / 2 \sigma^{2}} \times \mathrm{e}^{j 2 \pi f_{0}\left(x \cos \theta_{i}+y \sin \theta_{i}\right)}
$$

where $f_{0}$ and $\sigma$ are respectively the central frequency and the standard deviation of the Gaussian envelope of the filter. As advocated in the initial method of Fleet and Jepson, the following parameters were used: $N=6, f_{0}=0.25, \theta_{i}=i \frac{\pi}{N}$ and $\sigma=3$. To ensure sufficient confidence in the measure of OF vector estimates, i.e. to avoid unreliable measurements, a constraint on the estimated velocities was added. Using the matrix notations, singularity and round off errors in $A^{T} W A$ inversion (equation 4) can be avoided by testing the eigenvalues. The settings were defined as proposed by Bruno and Pellerin [21] ( $\lambda_{2} / \lambda_{1} \leq \varepsilon$, where $\lambda_{2}$ is the smaller eigenvalue and the threshold $\varepsilon$ is set to 0.01 ). We used this value with synthetic images, whereas we have empirically fixed the constraint $\mathcal{E}$ equal to 0.5 for the MR images. This ensures a sufficient $\mathrm{OF}$ density in the MR images. To summarize, the main differences between the method proposed by Bruno and Pellerin and the MPOF algorithm are the values of the spatial frequency $f_{0}$ and of the constraint $\mathcal{E}$. The settings of these parameters were best adapted to the MR images.

\section{METHOD EVALUATION}

\section{A. Synthetic Data}

The two optical flow algorithms have previously been evaluated on standard test sequences [4] for optical flow computation. Although there are available mathematical models of cardiac deformation [26], [27] (often obtained from tagged-MRI), they are relatively complex and difficult to apply in our case. Therefore, we preferred to create a synthetic image sequence reflecting basic motions of contraction and dilation. As the method we developed uses 2D Gabor filters [21], and as optical flow methods estimate the displacement vector fields without any a priori knowledge of the movement that occurs in the image sequence, the test sequence consisted in a simple $2 \mathrm{D}$ disk. The dilating and the contracting phases were equal in duration. This sequence was performed using the computer graphics software Blender ${ }^{l}$ (Fig. 1).

The image sequence consisted of 30 images, included two phases, the expansion phase of a 2D disk followed by its contraction. The disk radius evolves linearly through time (one pixel per frame). The disk has slightly varying grey level pixels within an image. The advantage of dealing with synthetic images is that the motion field is known and can be compared with the estimated flow field.

Moreover, the main advantage of the OF techniques is that it does not rely on any model to detect motion in image sequences.

\section{B. MRI Protocol}

Cine-MR images were acquired using breathhold retrospective ECG-gated SSFP-type sequences on a 3T imager (Trio TIM, Siemens Medical Solution, Germany) using a dedicated

\footnotetext{
${ }^{1}$ http://www.blender.org/
} 


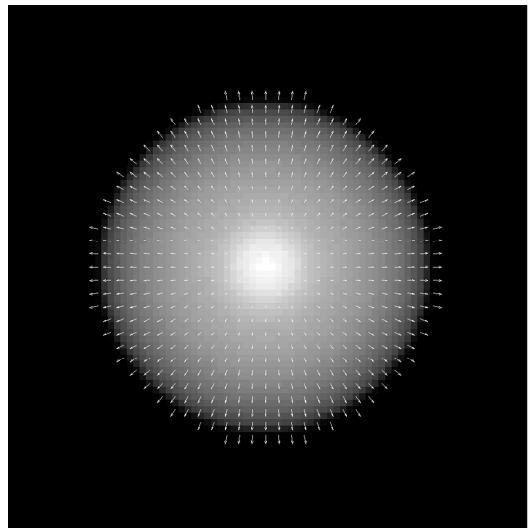

(a)

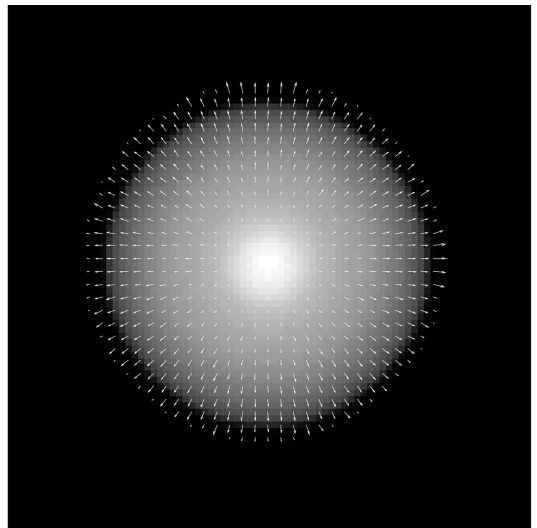

(b)

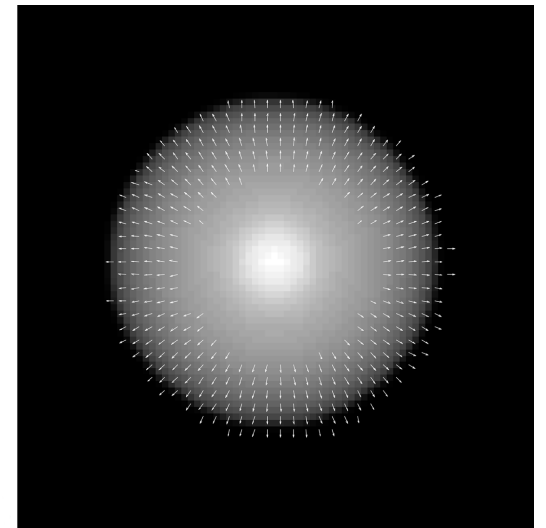

(c)

Fig. 1. Optical flow is displayed on the synthetic image sequence during the expansion of the 2D disk with (a) the correct motion (b) the IOF method (50 iterations were necessarv and the constraint on the gradient magnitude was $\|\Delta\|>1$ ). and with c) the MPOF method. cardiovascular 8-channel surface-coil array. Parallel imaging with the GRAPPA algorithm was applied with an acceleration factor of $\mathrm{R}=2$. Images from 11 representative normal hearts (4 men, 7 women, mean age: $39 \pm 17$ years, mean ejection fraction measured from MRI: $62 \pm 7 \%$ ) and one heart presenting a severe parietal dyssynchrony were used to evaluate our method and acquisitions along the short- and long-axis orientations were considered. Nominal acquisition parameters included: $\mathrm{TR} / \mathrm{TE}=2.51 \mathrm{~ms} / 1.84 \mathrm{~ms}, 50^{\circ} \mathrm{flip}$ angle, $6 \mathrm{~mm}$ slice thickness, 60 phases/cardiac cycle, breathhold duration of 15-17 s corresponding to 20-25 heartbeats, acquisition matrices (respectively, the pixel sizes) were $198 \times 288$ and $256 \times 224$ for the short- and long-axis orientations (respectively $1.0 \times 1.0$ and $1.2 \times 1.2 \mathrm{~mm}^{2}$ ) with rectangular field of view and temporal resolution of 10-15 ms/frame. The acquisition matrices were not interpolated. These images were then built using the manufacturer's reconstruction algorithm used on our MRI scanner.

\section{Evaluation}

The IOF (method of Horn and Shunck, and defined as the reference method) and the MPOF techniques have initially been tested on a synthetic image sequence. The aim was to investigate the behavior of the MPOF method and the IOF method when confronted with specific MRI characteristics such as brightness variations and noise.

The quantitative analysis of the cardiac wall motion from real MRI data was performed by tracking points of interest from different myocardial segments over time. Time-radial velocity curves $(\mathrm{cm} / \mathrm{s})$ as well as absolute displacement curves $(\mathrm{mm})$ were then computed. The analysis of the different curves allowed us to extract parameters reflecting the temporal delay between two myocardial segments at the early systole from the velocity curves and at the end of the systole from the displacement curves.

\section{Metrics for the optical flow evaluation}

The evaluation of the OF performance was carried out using the computation of the Angular Error (AE) [4],[5]. The AE between the correct velocity $\left(u_{c}, v_{c}\right)$ and an estimated one

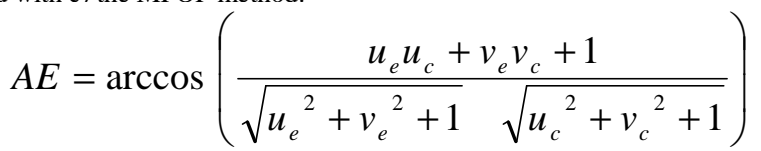

This provides the error (expressed in degrees) between the true and estimated motion at each pixel location with respect to the velocity modulus and orientation errors. The density of the OF was also evaluated on each image as the percentage of nonzero velocity vectors within the image. The mean $\mathrm{AE}$ refers to the mean angular error for a given OF density that is to say the mean $\mathrm{AE}$ for non-zero velocity estimates. All the images of the sequence were considered for the calculation of the mean $\mathrm{AE}$ and the $\mathrm{OF}$ density.

All data were expressed as mean \pm standard deviation of the AE. Statistical analysis was performed by a $t$-test to evaluate if the difference between two optical flow methods was significant. $p<0.05$ was considered statistically significant.

\section{Settings of the tested OF approaches}

Motion estimation was achieved using the classical OF methods of Horn and Shunck based on pixel intensity [18]. Settings of this method are similar to the original algorithm with at most 100 iterations, and motion is computed between two consecutive images. Furthermore, as mentioned by Horn and Schunck [18], and proposed by Barron et al. [4], an additional constraint on the magnitude of the local gradient

$(\|\nabla I\| \geq 1.0)$ ensures that there is sufficient local information for a reliable motion estimation. These settings define the IOF method. Motion estimation was also achieved using the method of Fleet and Jepson based on phase information with 3D filtering (referenced as the POF method) [5]. To compute the motion field with the POF method, the corresponding program implemented by Barron is utilized. The developed method, dedicated to MR images, is called MPOF and is a modified version of the POF technique with $2 \mathrm{D}$ filtering. In this study we then considered the IOF approach as being the reference method. $\left(u_{e}, v_{e}\right)$ is equal to: 
Robustness against noise and brightness variations of the OF methods

In order to evaluate the robustness and the sensitivity of the methods, the mean $\mathrm{AE}$ was also computed in the presence of Rician noise or brightness variations on the synthetic image sequence. Rician noise is characterized by its standard deviation $\sigma$. So, the images were corrupted with a Rician noise whose standard deviation varied from 1 to 20 (the last value corresponds to highly corrupted images). The brightness variation is the pixel intensity variation expressed in percentage between two consecutive images. For this test, the mean $\mathrm{AE}$ was evaluated when global brightness variation on pixel intensities occurred from 1 to $16 \%$ (increasing levels: from low to high brightness variations).

Moreover, the noise removal algorithm proposed by McGibney and Smith was compared with a Gaussian filtering on two synthetic images corrupted with a Rician noise, the first with a standard deviation equal to 10 and the second with a standard deviation equal to 20 . The mean square error (MSE) was calculated between the original and the filtered images.

\section{Measurement of the heart wall displacement}

A visual evaluation of the OF results on MR images (color coding was used on these images) allows one to verify whether the estimated motion field is coherent with the heart behavior during a cardiac cycle. This is, however, not sufficient and a quantitative analysis of the results is also necessary. On the first image of the series, four points of interest of the myocardium close to the endocardium are located at the anterior, septal, posterior and lateral areas. The

TABLE I

OPTICAL FLOW (OF) RESULTS FOR DIFFERENT TECHNIQUES APPLIED ON THE SYNTHETIC SEQUENCE

\begin{tabular}{lcr}
\hline \multicolumn{1}{c}{ Technique } & $\begin{array}{l}\text { OF } \\
\text { Density }\end{array}$ & Mean AE \\
\hline Horn and Schunck & $100 \%$ & $12.6^{\circ} \pm 11.4^{\circ}$ \\
IOF (Referenced method) $*$ & $55 \%$ & $4.9^{\circ} \pm 6.7^{\circ}$ \\
POF* & $13 \%$ & $4.5^{\circ} \pm 8.5^{\circ}$ \\
MPOF* & $52 \%$ & $5.7^{\circ} \pm 2.8^{\circ}$ \\
MPOF with $\left(\lambda_{2} / \lambda_{1} \leq 0.01\right)^{*}$ & $36 \%$ & $5.3^{\circ} \pm 3.0^{\circ}$
\end{tabular}

The mean angular error (AE) and its standard deviation are reported for a certain density (i.e. percentage of non-zero velocity estimates within the images). The theoretical OF density of the image is $48.1 \%$.

* No significant difference between the different methods.

position of these points from one frame to another is calculated according to the velocity vectors computed on each frame. The projection of the velocity vectors perpendicular to the endocardium is calculated thereby providing the velocity of the motion. For each point, the calculation of the associated velocity on each frame allows the creation of time-radial velocity curves. This analysis is performed on short-axis slices. From the time-radial velocity curve of the normal heart,

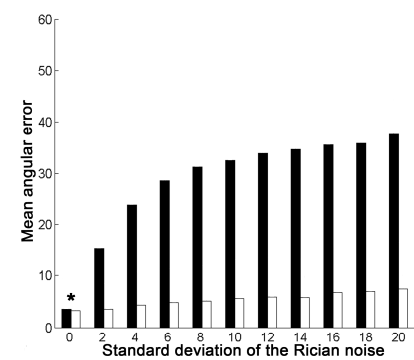

(a)

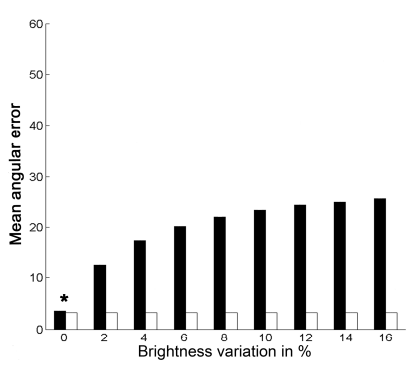

(b)
Fig. 2. Study of the angular error (AE, in degrees) of IOF and MPOF methods against Rician noise and brightness variations for the synthetic image sequence during contraction. The AE of the IOF and MPOF methods are respectively depicted by black and white bars. There is no significan difference between the IOF and MPOF methods when there is no noise and no brightness variation $\left(*: \mathrm{p}<10^{-5}\right)$.

Peak Systolic Velocity (PSV) and Peak Diastolic Velocity (PDV) are calculated and expressed as pixels per frame, but are converted into $\mathrm{cm} / \mathrm{s}$ with knowledge of spatial and temporal resolutions. The mean velocities were calculated for the 11 normal subjects. The temporal delay of 7 images (corresponding to a temporal support of 15 images [28]) required by the POF and MPOF techniques is overcome by the use of retrospective ECG-gated sequences: in this way it is possible to compute the vectors in the first and last images of a given sequence.

\section{RESUlTS}

\section{A. Synthetic sequence}

\section{Noiseless synthetic sequence}

Considering the original synthetic sequence, the estimated motion fields of the IOF and MPOF methods are depicted on Fig. 1. Vectors are orientated in the direction of the motion, i.e. in the outer disk for the dilating phase. Statistical results are presented in Table 1 according to the different techniques. For each technique, the mean and standard deviation of the AE according to the motion density were evaluated. The OF techniques give sub-pixel motion precision.

The regularization constraint of the IOF applied on a uniform background gives poor results (mean AE of 12.6 $\pm 11.4^{\circ}$ ) because motion is detected beyond the outer edge of the disk (i.e. on the black background), even in the absence of movement. To overcome this problem, the IOF method has a constraint on the gradient magnitude (as gradients are supposed to be null on a uniform background). As the motion is hardly ever detected on the background, the density of the estimated OF decreases (55\%) approaching the real motion density $(48 \%)$ and the mean $\mathrm{AE}$ is improved $\left(4.9^{\circ} \pm 6.7^{\circ}\right)$ when compared with the initial IOF method. The initial POF technique gives a mean $\mathrm{AE}$ of $4.5^{\circ} \pm 8.5^{\circ}$. The modified version of the POF algorithm (MPOF) using 2D spatial filtering gives statistical results close to the reference method (IOF) with a mean $\mathrm{AE}$ of $5.7^{\circ} \pm 2.8^{\circ}$. Finally, our method (MPOF) with the additional constraint to avoid unreliable measurements $\left(\lambda_{2} / \lambda_{1} \leq \varepsilon\right)$ has an $\mathrm{AE}$ of $5.3^{\circ} \pm 3.0^{\circ}$. This additional constraint not only decreased the estimated motion 

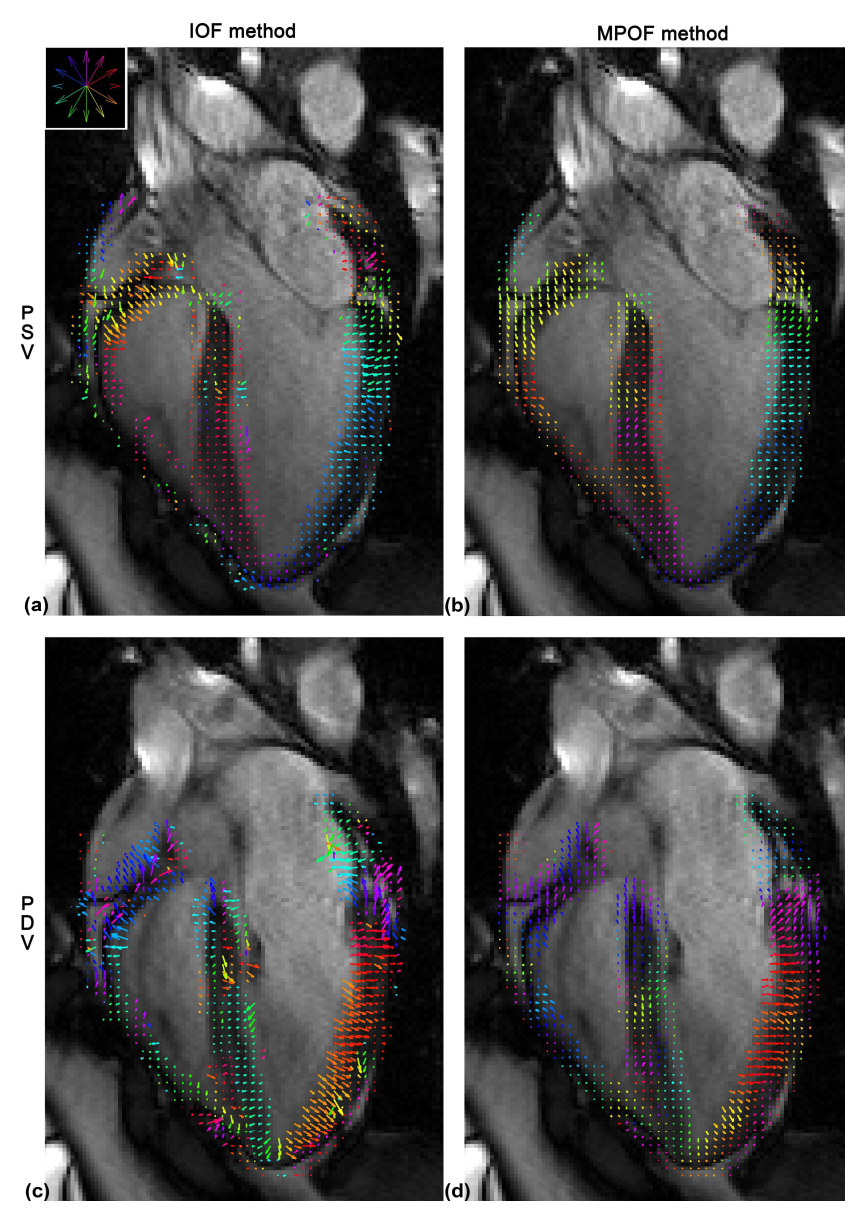

Fig. 3. OF estimates on the four-chamber MRI sequence for the first normal heart at PSV (on the first row) and PDV (on the second row) using either the IOF on the left (with the constraint on gradient magnitude set to $\|\Delta\| \geq 1$ ) or MPOF method on the right. The OF visualization was restricted to the myocardium area. A mask was applied in order to display the vector field only at the level of the myocardium.

density, but reduced the mean AE. This constraint is necessary in the case of a real image analysis to ensure sufficient confidence in the estimates. Finally, there are no significant differences between the IOF, the POF and MPOF methods $\left(\mathrm{p}<10^{-5}\right)$.

\section{Noisy synthetic sequence}

When corrupting the synthetic image sequence with Rician noise, the mean $\mathrm{AE}$ evolves differently according to the $\mathrm{OF}$ method (Fig. 2a): For the IOF technique, the greater the noise level, the more the detected motion was perturbed. This results in an important increase of the AE. The corresponding results for the MPOF technique are better. Moreover, when brightness variations occur, the quality of the IOF field deteriorates whereas the MPOF method does not seem to be altered (Fig. 2b). It should be noted that the mean AE of the IOF technique increases due to an overestimation of the velocity magnitudes although the movement orientation appears to be correct. More generally, and with reference to the two bar graphs on Fig. 2, the mean and standard deviation of the AE may appear abnormally high for the IOF method,
TABLE 2

MAGNITUDE OF THE ESTIMATED VELOCITIES (CM/S) CALCULATED ON 11 HEALTHY SUBJECTS USING THE 4-SEGMENTAL MODEL AT PSV (PEAK SYSTOLIC VELOCITY) AND PDV (PEAK DIASTOLIC VELOCITY).

\begin{tabular}{|c|c|c|}
\hline & PSV & PDV \\
\hline Anterior & $4.7 \pm 1.0$ & $4.0 \pm 1.0$ \\
\hline Lateral & $4.6 \pm 0.7$ & $5.0 \pm 1.0$ \\
\hline Posterior & $5.1 \pm 0.9$ & $6.1 \pm 1.5$ \\
\hline Septum & $2.9 \pm 0.5$ & $3.1 \pm 0.9$ \\
\hline
\end{tabular}

but it is the consequence of extensive tests on highly deteriorated images. By comparison, the standard deviation for the MPOF method is lower.

Concerning the noise filtering algorithm, the MSE was lower with the noise removal algorithm proposed by McGibney and Smith [25] than with the Gaussian filter (6.3 vs 14.2 for the synthetic image corrupted with a Rician noise with a standard deviation equal to 10 , and 17.2 vs 45.6 for the synthetic image corrupted with a Rician noise with a standard deviation equal to 20 ).

\section{B. Application to MRI}

To illustrate the two methods (IOF and MPOF) we present the results obtained on two representative cardiac MRI examinations from two normal hearts on the long-axis and short-axis orientations (Fig. 3 and 4).

\section{Visual interpretation of the velocity vectors}

The visual evaluation of the estimated flow field of the IOF and MPOF throughout the MRI sequences shows that when the POFCE is respected, such as on the myocardial areas on the long-axis images of the first examination (Fig. 3), the estimated motion field is quite spatially homogeneous and similar when using either the IOF (Fig. 3a and 3c) or the MPOF techniques (Fig. $3 b$ and $3 d$ ). The global motion estimated within the images, using these two OF techniques, was faithful to normal heart behavior and the estimated flow field was consistent with the direction of the movement (myocardial contraction and relaxation). There is enough texture information on the myocardium of the short axis images to detect the rotational component of motion due to the left ventricular twist at the early systole.

\section{Velocity measurements}

Table 2 gives the velocities PSV and PDV obtained on the 11 healthy subjects with the MPOF method at each myocardial segment on a 4-segmental model.

Fig. 5 and Fig. 6 present the evolution of the absolute displacement and the radial velocity of the septal and lateral segments on the short-axis orientations along the entire cardiac cycle of respectively, the first normal heart and a heart presenting a severe parietal dyssynchrony. The temporal delays $(\Delta t)$ measured from the displacement curves at the end of the systole are respectively $79 \mathrm{~ms}$ (Fig. 5a) and $305 \mathrm{~ms}$ (Fig. 6a) for the normal and desynchronized heart. The temporal delays measured from the radial velocity curves at the early systole are $21 \mathrm{~ms}$ (Fig. 5b) and $106 \mathrm{~ms}$ (Fig. 6b).

For one normal case, we have compared the absolute displacement measurements obtained with MPOF method on cine-MR images on a short-axis basal slice with the results 
obtained with the inTag software [29] applied on tagged MRI on the same slice. Although the values are slightly higher with the MPOF method on cine-MR images, we can say on this example, that the results are very close between the two approaches for the anterior wall $(6.3 \mathrm{~mm}$ vs $5.2 \mathrm{~mm})$, lateral wall $(7.4 \mathrm{~mm}$ vs $7.4 \mathrm{~mm})$, posterior wall $(6.8 \mathrm{~mm}$ vs $6.4 \mathrm{~mm})$ and the septum (4.8 $\mathrm{mm}$ vs $4.4 \mathrm{~mm}$ )."

Image quality: Brightness variation, partial volume effect, blood inflow
Considering the short-axis sequence of the first examination, the through plane motion of the heart at the very basal part of the heart may cause brightness variations and partial volume effects. This will degrade the results of the IOF by overestimating the myocardial motion field. PDV at the lateral and posterior walls of, respectively, 8.3 and $15.2 \mathrm{~cm} / \mathrm{s}$ were found (Fig. 4c). This behavior has already been observed during the testing of the IOF method on the synthetic image sequence when confronted with brightness variations. This is due to the failure to comply with the OFCE that implies
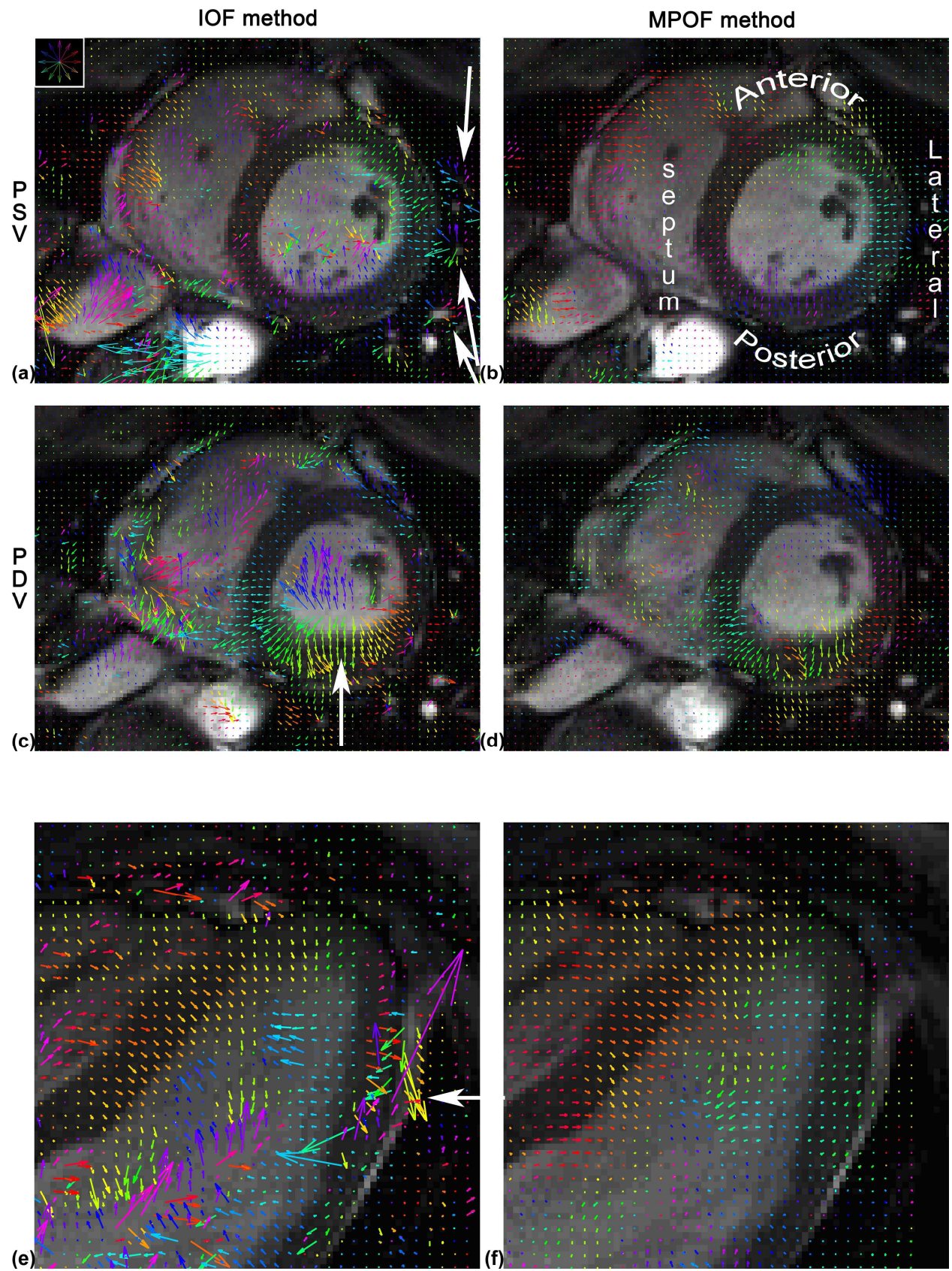

Fig. 4. OF estimates on the short-axis MRI sequence for the first normal heart (a-d) and on the long-axis orientation for the second normal heart (e-f). For the first sequence, motion is displayed at (a-b) PSV and (c-d) PDV. For the second sequence, motion is depicted during early systole. Two OF techniques are used, the IOF on the left (with the constraint on gradient magnitude set to $\|\Delta\| \geq 1$ ) and MPOF method on the right. Considering the IOF technique, the white arrows depict (a) motion singularities due to blood inflow through vessels, (c) overestimated movement due to important through-plane motion and (e) poorer results due to the through-plane incoming of fat tissues. 
brightness consistency. With regards to the long-axis sequence (Fig. 3c) of the same examination, the OFCE is better respected and thus the results between the two OF methods are closer. Moreover, in the presence of partial volume effects (Fig. 4c and 4d) on the inferoseptal and inferior LV segments, especially when coupled with low tissue contrast, the IOF technique gives poorer results (Fig. 4c) compared with the MPOF method (Fig. 4d).

The left and right cavities are subject to blood flow disturbances causing inconsistent motion detection when using the IOF method. This is particularly visible at PDV along the short-axis orientations (Fig. 4c). However, this will not affect the motion analysis at the level of the heart wall: the estimated motion field at the level of the endocardium is homogeneous and concords with the myocardial relaxation movement.

Moreover, small vessels close to the inferolateral heart segment on the short-axis images (Fig. 4a) present motion singularities. They are induced by blood inflow through those vessels that alters the quality of the velocity flow field given by the IOF method (Fig. 4a). With the MPOF method, the singularities are discarded by the confidence measure constraint.

Finally, on the examination of the second normal heart, the through-plane incoming of fat tissues (corresponding to the appearance of an hyper-intense signal on Fig. 4e and 4f), close to the epicardium on the apicolateral LV segment, led to an absurd motion field for the IOF method (Fig. 4e). This was not the case with the MPOF technique.

\section{DISCUSSION}

In this paper we have presented an adapted optical flow approach to extract local myocardial motion directly from cine-MRI sequences. Although the study of global myocardial function is widely assessed using cardiac cine-MRI [1], [30], the study of local contractile function to detect movement abnormalities with MRI necessitates post-processing and thus, is not commonly used in clinical practice.

The available techniques to assess local motion include tagged MRI [29], [31]-[33], displacement encoding sequence (DENSE) [34] and velocity-encoded phase contrast MRI [35]. Among these techniques, tagged MRI is generally the most utilized [1]. This technique gives information on heart wall displacements by following the distortion of tag lines throughout the cardiac cycle. The detection and tracking of tag lines through the image sequence is not straightforward [36] and necessitate the use of dedicated software [29], [37], [38]. This kind of analysis allows the study of heart tag deformation by measuring the myocardial strain and torsion [39],[40]. However, even if local motion information is well assessed with myocardial tagged MRI, it is noteworthy that the quality of the results depends on tag persistence, spacing and thickness. Besides, tagged MRI cannot entirely replace cineMRI for estimating EF and myocardial thickness and requires an additional acquisition.

The study of cardiac wall motion in terms of LV dyssynchrony is also available with echocardiography [41] using different modalities: M-mode, TDI (Tissue Doppler
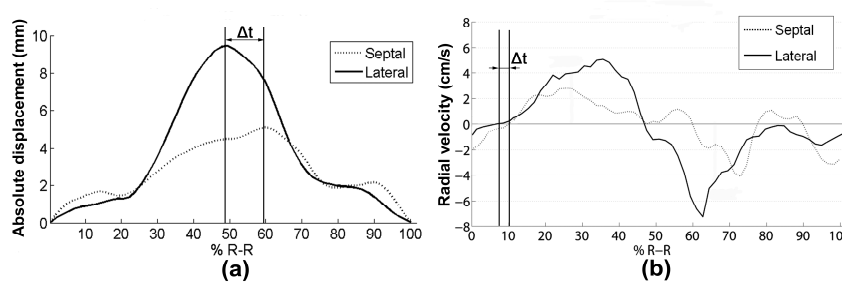

Fig. 5. (a) Absolute displacement and (b) radial velocity curves along the cardiac cycle expressed as a percentage of the R-R interval (719 ms) according to the septal and lateral segments of the short-axis MRI sequence of the normal heart examination. $\Delta t$ is the temporal delay. The MPOF method was used to estimate the motion vector field. The LV ejection fraction computed from MRI was $72 \%$. The PSV and the PDV appear at respectively $27 \%$ and $66 \%$ of R-R duration.
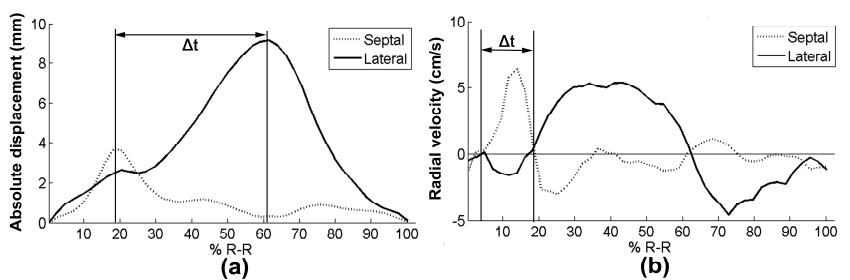

Fig. 6. (a) Absolute displacement and (b) radial velocity curves along the cardiac cycle expressed as a percentage of the R-R interval (726 ms) according to the septal and lateral segments of a short-axis MRI sequence of a patient with intraventricular dyssynchrony. $\Delta t$ is the temporal delay. The MPOF method was used to estimate the motion vector field. The LV ejection fraction computed from MRI was $31 \%$.

Imaging), myocardial deformation imaging (based on speckle tracking) and 2D and 3D echocardiography.

The benefits of optical flow techniques are two-fold. Firstly, no further MRI acquisitions are necessary. The limitation of MRI examination duration improves patient comfort and image quality by reducing the number of breathholds. The second benefit is that motion information naturally present in classical cine-MRI is utilized, thus taking advantage of the excellent spatio-temporal resolution provided by SSFP sequences. Concerning the quality of the proposed MPOF method, the estimated velocities are globally similar with values obtained in other studies with echocardiography or MRI. In particular, using the Tissue Doppler Imaging (TDI) modality, Miyatake et al. [15] found a velocity of 5.1 \pm 1.0 $\mathrm{cm} / \mathrm{s}$ at the LV posterior wall on 7 subjects who did not present cardiac disease. Bussadori et al. found on 30 healthy subjects a mean peak systolic velocity of $4.7 \pm 1.2 \mathrm{~cm} / \mathrm{s}$ for the lateral wall and of $4.4 \pm 1.1 \mathrm{~cm} / \mathrm{s}$ in the septal wall, and a mean peak diastolic velocity of $6.2 \pm 1.7 \mathrm{~cm} / \mathrm{s}$ for the lateral wall and of $5.2 \pm 1.8 \mathrm{~cm} / \mathrm{s}$ in the septal wall [13]. These values are slightly higher than ours, except for the peak systolic velocity in the lateral wall. On ten patients without evidence of prior infarction, Karwatowski et al. have found a mean velocity of $5.6 \pm 1.7 \mathrm{~cm} / \mathrm{s}$ for all segments from MR images [16]. However, in a previous study with MR velocity mapping in 31 healthy subjects [42], at the level of the LV posterior wall during early diastole, the mean measured velocity was 9 $\pm 3 \mathrm{~cm} / \mathrm{s}$. The mean corresponding velocity estimate with our MPOF method for the normal hearts was lower $(6.1 \pm 1.5$ $\mathrm{cm} / \mathrm{s})$. Maret et al. have found on 13 patients a mean radial value of $3.74 \mathrm{~cm} / \mathrm{s}$ from long-axis cine-MRI [43]. The variation in the results can be explained by the chosen 
technique or the studied population. Moreover, the synthetic sequence shows that the MPOF method is relatively more robust to brightness variations and noise than the IOF approach.

OF techniques necessitate taking into account both the MR image properties and the $\mathrm{OF}$ technique requirements. The $\mathrm{OF}$ estimation requires a sufficient temporal sampling that ensures small displacements of the structures between two consecutive images. This is partly performed by tuning MRI acquisition parameters such as temporal resolution and pixel size, but is not always sufficient. The MPOF algorithm is set to precisely detect motion from 0 to 1.25 pixel/frame (the velocity must be less than the half-period of the highest spatial frequency [44]), although the heart wall displacements can exceed this threshold. According to the acquisition setting (temporal resolution and pixel size) and heart behavior, the use of a Gaussian low-pass pyramidal image decomposition [22] is necessary to extend the domain of the allowed velocities. The choice of the acquisition plane orientation is assumed to be free because the OF technique does not need prior information on the image content. Regarding the noise removal algorithm, the signal-to-background contrast is more faithfully restored, improving the gradient estimation in this area. In this way, when considering MR examinations, gradient estimation is well performed in areas close to the air-tissue and in particular epicardium-lung interface. Finally, the 3T SSFP sequence is subject to off-resonance or banding artifacts that cannot be fully avoided, but may be moved out of the current ROI for cardiac MRI [45]. This prevents incorrect motion detection on the myocardium. Compared with data acquired at $1.5 \mathrm{~T}$, the use of a $3 \mathrm{~T}$ magnetic field improves the SNR in the images. However, the use of parallel imaging introduces a drop in SNR [46]. The increase in baseline SNR for 3.0 T imaging overcompensated for the SNR reduction from parallel imaging. Thus, the gain is principally a time reduction in the data acquisition. Therefore the results obtained at $3.0 \mathrm{~T}$ with parallel imaging should be the same as those obtained at $1.5 \mathrm{~T}$ without parallel imaging. Although MPOF seems to provide interesting results when applied to cardiac MR images, some limitations appear. Indeed, as working on the phase information of the signal is more stable (compared with the signal intensity), MPOF better succeeds in estimating motion. However, the high computational time (around one hour on a conventional microcomputer to estimate the motion on 60 images), the difficulty to respect the POFCE in the event of poor quality images and the difficulty to validate the technique in absence of a gold standard for MRI remain limitations. Optical flow algorithms are generally either very accurate and slow, or very fast and inaccurate. For diagnostic purposes, it is better to focus on accurate algorithms such as frequency-based methods, knowing that many researchers are working on hardware implementations in order to accelerate results [47].

\section{CONCLUSION}

In conclusion, 2D optical flow applied to cardiac SSFP-type cine-MRI examinations gives promising results. In conjunction with cardiac cine-MRI, OF techniques may help in detecting local wall motion abnormalities and measuring their velocity in each studied pixel. From local motion information, regional motion parameters can be determined, giving displacements and velocities per myocardial segment along the cardiac cycle.

One potential perspective of this approach would be the quantification of LV mechanical dyssynchrony. The mechanical dyssynchrony could be assessed from the timeradial velocity curves according to each myocardial segment by estimating the temporal delay between the curves. In this particular pathology, an estimation of the peak systolic velocities for each segment would provide an accurate estimation of the dyssynchrony. Indeed, it is well known that the peak velocities will not occur at the same time in the event of mechanical dyssynchrony.

\section{ACKNOWLEDGMENT}

The authors thank Dr. Jean-Christophe Eicher (University Hospital of Dijon, France) and Dr. Sophiane Houamria (University Hospital of Dijon, France) for their expertise in cardiology.

\section{REFERENCES}

[1] F. H. Epstein, "MRI of left ventricular function," J. Nucl. Cardiol., vol. 14, no. 5, pp. 729-744, 2007.

[2] D. J. Pennell, U. P. Sechtem, C. B. Higgins, W. J. Manning, G. M. Pohost, F. E. Rademakers, A. C. van Rossum, L. J. Shaw, K. E. Yucel, "Clinical indications for cardiovascular magnetic resonance (CMR): Consensus Panel report," Eur. Heart J,. vol. 25, no. 21, pp. 1940-1965, Nov. 2004.

[3]X. Papademetris, A. J. Sinusas, D. P. Dione, R. T. Constable, J. S. Duncan, "Estimation of 3-D left ventricular deformation from medical images using biomechanical models," IEEE Trans. Med. Imaging, vol. 21, no. 7, pp. 786800, Jul. 2002

[4] J. L. Barron, D. J. Fleet, S. S. Beauchemin, "Performance of optical flow techniques," Int. J. Comput. Vision, pp. 43-77, 1994.

[5] D. J. Fleet, A. D. Jepson, "Computation of component image velocity from local phase information," Int. J. Comput. Vision, pp. 77-104, 1990.

[6] S. C. Amartur, H. J. Vesselle, "A new approach to study cardiac motion: the optical flow of cine MR images," Magn. Reson. Med., vol. 29, no.1, pp 5967, Jan. 1993.

[7] A. A. Amini, J. Prince, "Measurement of Cardiac Deformation from MRI: Physical and Mathematical Models," Dordrecht: Kluwer Academic Publishers, 2001.

[8] J. L. Barron, "Experience with 3D Optical Flow on Gated MRI Cardiac Datasets," in CRV04, 2004, pp. 370-377.

[9] T. Arts, F. W. Prinzen, T. Delhaas, J. R. Milles, A. C. Rossi, P. Clarysse, "Mapping displacement and deformation of the heart with local sine-wave modeling," IEEE Trans. Med. Imaging, vol. 29, no. 5, pp. 1114-1123, May 2010.

[10] L. Dougherty, J. C. Asmuth, A. S. Blom, L. Axel, R. Kumar, "Validation of an optical flow method for tag displacement estimation," IEEE Trans. Med. Imaging, vol. 18, no. 4, pp. 359-363, Apr. 1999.

[11] J.L. Prince, E.R. McVeigh, "Motion estimation from tagged MR image sequences," IEEE Trans. Med. Imaging, vol. 11, no. 2, pp. 238-249, 1992.

[12] S. M. Song, R. M. Leahy, "Computation of 3-D velocity fields from 3-D cine CT images of a human heart," IEEE Trans. Med. Imaging, vol. 10, no. 3, pp. 295-306, 1991

[13] C. Bussadori, A. Moreo, M. Di Donato, B. De Chiara, D. Negura, E. Dall'Aglio, E. Lobiati, M. Chessa, C. Arcidiacono, J. S. Dua, F. Mauri, M. Carminati, "A new 2D-based method for myocardial velocity strain and strain rate quantification in a normal adult and paediatric population: assessment of reference values," Cardiovasc. Ultrasound, vol. 7:8, Feb. 2009.

[14] M. Sühling, M. Arigovindan, C. Jansen, P. Hunziker, M. Unser, "Myocardial motion analysis from B-mode echocardiograms," IEEE Trans. Image Process., vol. 14, no. 4, pp. 525-536, Apr. 2005.

[15] K. Miyatake, M. Yamagishi, N. Tanaka, M. Uematsu, N. Yamazaki, Y. Mine, A. Sano, M. Hirama, "New method for evaluating left ventricular wall 
motion by color-coded tissue Doppler imaging: in vitro and in vivo studies," J. Am. Coll. Cardiol., vol. 25, no. 3, pp. 717-724, Mar. 1995.

[16] S.P. Karwatowski, S.J.D. Brecker, G.Z. Yang, D.N. Firmin, M. St John Sutton, S.R. Underwood, "A comparison of left ventricular myocardial velocity in diastole measured by magnetic resonance imaging and left ventricular filling measured by Doppler echocardiography," Eur Heart J, vol. 17 , no. 5, pp. 795-802, May 1996

[17] H. Liu, T.-H. Hong, M. Herman, R. Chellappa, "Accuracy vs. Efficiency Trade-offs in Optical Flow Algorithms," Comp. Vis. Image Und., vol. 72, no. 3, pp. 271-286, Dec. 1998.

[18] B. K. P. Horn, B. G. Schunck, "Determining Optical Flow", Artif. Intell., vol. 17, pp.185-203, 1981.

[19] B. D. Lucas, T. Kanade, "An iterative image registration technique with an application to stereo vision," in IJCAI81, 1981, pp 674-679.

[20] D. J. Heeger, "Optical Flow Using Spatiotemporal Filters," in ICCV87, 1987 , pp. $181-190$.

[21] E. Bruno, D. Pellerin, "Robust motion estimation using spatial Gabor-like filters," Signal Process., vol. 82, pp. 297-309, 2002

[22] A. Chéhikian, "Optimal algorithms for low-pass and Laplacian image pyramids computation," Traitement du Signal, vol. 9, pp. 297-307, 1992.

[23] H. Gudbjartsson, S. Patz, "The Rician Distribution of noisy MRI Data," Magn. Reson. Med., vol. 34, no. 6, pp. 910-914, Dec. 1995.

[24] O. Dietrich, J. G. Raya, S. B. Reeder, M. Ingrisch, M. F. Reiser, S.O Schoenberg, "Influence of multichannel combination, parallel imaging and other reconstruction techniques on MRI noise characteristics," Magn. Reson. Imaging, vol. 26, no. 6, pp.754-762, Jul. 2008

[25] G. McGibney, M. R. Smith, "An unbiased signal-to-noise ratio measure for magnetic resonance images," Med. Phys., vol. 20, no. 4, pp. 1077-1078, Jul.-Aug. 1993

[26] T. Arts, W. C. Hunter, A. Douglas, A. M. Muijtjens, R. S. Reneman, "Description of the deformation of the left ventricle by a kinematic model," Journal of biomechanics, vol. 25, pp. 1119-1127, 1992.

[27] E. Waks, J. Prince, A. Douglas, "Cardiac Motion Simulator for Tagged MRI," In MMBIA '96, 1996, pp 182-191.

[28] D.J. Fleet, K. Langley, "Recursive filters for optical flow", IEEE Trans. Pattern Anal. Mach. Intell., vol. 17, no 1, pp. 61-67, 1995.

[29] T. Arts, F. W. Prinzen, T. Delhaas, J. R. Milles, A. C. Rossi, P. Clarysse, "Mapping Displacement and Deformation of the Heart With Local Sine-Wave Modeling," IEEE Trans. Med. Imaging, vol. 29, no. 5, pp. 1114 - 1123, 2010.

[30] E. R. McVeigh, "MRI of myocardial function: motion tracking techniques," Magn. Reson. Imaging, vol. 14, no. 2, pp. 137-150, 1996.

[31] L. Axel, L. Dougherty, "MR imaging of motion with spatial modulation of magnetization," Radiology, vol. 171, no. 3, pp. 841-845, Jun. 1989.

[32] E. A. Zerhouni, D. M. Parish, W. J. Rogers, A. Yang, E. P. Shapiro, "Human heart: tagging with MR imaging-a method for noninvasive assessment of myocardial motion," Radiology, vol. 169, no.1, pp. 59-63, Oct. 1988.

[33] N. F. Osman, W. S. Kerwin, E. R. McVeigh, J. L. Prince, "Cardiac motion tracking using CINE harmonic phase (HARP) magnetic resonance imaging," Magn. Reson. Med., vol. 42, no. 6, pp. 1048-1060, Dec. 1999.

[34] A. H. Aletras, S. Ding, R. S. Balaban, H. Wen, "DENSE: displacement encoding with stimulated echoes in cardiac functional MRI," J. Magn. Reson., vol. 137, no. 1, pp. 247-252, Mar. 1999.

[35] M. Markl, B. Schneider, J. Hennig, "Fast phase contrast cardiac magnetic resonance imaging: improved assessment and analysis of left ventricular wall motion," J. Magn. Reson. Imaging, vol. 15, no. 6, pp. 642-653, Jun. 2002.

[36] J. P. Finn, K. Nael, V. Deshpande, O. Ratib, G. Laub, "Cardiac MR imaging: state of the technology," Radiology, vol. 241, no. 2, pp. 338-354 Nov. 2006.

[37] L. Axel, R. C Goncalves, D. Bloomgarden, "Regional heart wall motion: two-dimensional analysis and functional imaging with MR imaging," Radiology, vol. 183, no. 3, pp. 745-750, Jun. 1992.

[38] M. A. Guttman, J. L. Prince, E. R. McVeigh, "Tag and contour detection in tagged MR images of the left ventricle," IEEE Trans. Med. Imaging, vol. 13, no. 1, pp. 74-88, 1994.

[39] M. J. Götte, T. Germans, I. K. Russel, J. J. Zwanenburg, J. T. Marcus, A C. van Rossum, D. J. van Veldhuisen, "Myocardial strain and torsion quantified by cardiovascular magnetic resonance tissue tagging: studies in normal and impaired left ventricular function," J. Am. Coll. Cardiol., vol. 48 , no. 10, pp. 2002-2011, Nov. 2006.

[40] K. N. Hor, W. M. Gottliebson, C. Carson, E. Wash, J. Cnota, R. Fleck, J. Wansapura, P. Klimeczek, H. R. Al-Khalidi, E. S. Chung, D. W. Benson, and W. Mazur, "Comparison of magnetic resonance feature tracking for strain calculation with harmonic phase imaging analysis," JACC Cardiovasc Imaging, vol. 3, no. 2, pp. 144-51, Feb 2010.
[41] S. Kapetanakis, A. Bhan, M. J. Monaghan, "Echo determinants of dyssynchrony (atrioventricular and inter- and intraventricular) and predictors of response to cardiac resynchronization therapy," Echocardiography, vol. 25, no. 9 , pp. $1020-1030$, Oct. 2008

[42] S. P. Karwatowski, R. Mohiaddin, G. Z. Yang, D. N. Firmin, M. S. Sutton, S. R. Underwood, D. B. Longmore, "Assessment of regional left ventricular long-axis motion with MR velocity mapping in healthy subjects," J. Magn. Reson. Imaging, vol.4, no. 2, pp. 151-155, Mar.-Apr. 1994.

[43] E. Maret, T. Todt, L. Brudin, E. Nylander, E. Swahn, J. L. Ohlsson, and J. E. Engvall, "Functional measurements based on feature tracking of cine magnetic resonance images identify left ventricular segments with myocardial scar," Cardiovasc Ultrasound, vol. 7, p. 53, 2009.

[44] S. F. Wu, J. Kittler, "A differential method for simultaneous estimation of rotation, change of scale and translation," Signal Process Image Commun, vol. 2, no. 1, pp. 69-80, May 1990.

[45] M. Schar, S. Kozerke, S. E. Fischer, P. Boesiger, "Cardiac SSFP imaging at 3 Tesla" Magn. Reson. Med., vol. 51, no. 4, pp. 799-806, Apr. 2004.

[46] O.Wieben, C. Francois, S.B. Reeder, "Cardiac MRI of ischemic heart disease at 3T: Potential and challenges," Eur J Radiol, vol. 65, no. 1, pp. 1528 , jan. 2008.

[47] Y. Nagy, M. Saeb, Y. El-Sonbaty, "VHDL-Based Simulation of a Parallel Implementation of a Phase-Based Algorithm for Optical Flow," In AVSS '06 IEEE International Conference on Video and Signal Based Surveillance, 2006, pp. 27.

Marie Xavier received his Ph.D. in Informatics and Image Processing from the University of Burgundy at Dijon (France) in 2010. She is currently working in the "Laboratoire d'Electronique, Informatique et Image (LE2I)" of the University of Burgundy, until 2006. Her research interests are in image processing, especially in medical imaging. Her works cover the study of the moving organs, and the flow quantification in the aorta.

Alain Lalande received his Ph.D. in Biophysics and Medical Image processing from the University of Burgundy at Dijon (France) in 1999. Since 2000, he is an Assistant Professor of the medical image processing group at the "Laboratoire d'Electronique, Informatique et Image (LE2I)" of the University of Burgundy. His research interests concern the imaging of the cardio-vascular system (in particular MRI), and cover the topics of image acquisition, image post-processing and protocol design. His works cover the automatic study of the myocardial viability (in the case of ischemic pathologies), the evaluation of the cardiac wall motion (in the case of myocardial asynchronism) and the functional study of the aorta (wall stress evaluation, elasticity evaluation, flow quantification, etc.).

Paul M. Walker received the B.Sc. (first class) degree in applied physics from Aston University in Birmingham, England in 1984 and the Ph.D. degree in nuclear magnetic resonance imaging from the Postgraduate Medical School, Hammersmith Hospital, University of London, England in 1988. He is currently an Assistant Professor in the Medical Imaging Group at the "Laboratoire d'Electronique, Informatique et Image (LE2I)" of the University of Burgundy. His current research interests include MR imaging and MR spectroscopy in cancer and cardiovascular domains.

François Brunotte received his MD in 1984 from Nancy I University, Nancy, France. He is a professor of nuclear medicine at the university of Burgundy at Dijon since 1991. He is head of the joint nuclear medicine and Magnetic Resonance Spectroscopy department of the University Hospital and of the Cancer Center. $\mathrm{He}$ is member of the "Laboratoire d'Electronique, Informatique et Image (LE2I)" of the University of Burgundy. His research interests regard cardiovascular imaging especially in coronary heart disease and cancer imaging.

Louis Legrand received his Doctorate degree in electrical engineering in 1982, and his Doctorate in Sciences degree (Accreditation to supervise research) in 1989, both from the Nancy I University, Nancy, France. Since 1983, Dr. Legrand is with the University of Burgundy. He is currently a member of the medical image processing group at the "Laboratoire d'Electronique, Informatique et Image (LE2I)". His research interests are in medical image processing, especially of moving organs, and in human motion. 